\title{
Estrategias narrativas audiovisuales de desinformación en YouTube de la nueva extrema derecha europea
}

\section{Audiovisual narrative strategies of disinformation on YouTube of the new European far right}

\author{
Aarón Rodríguez-Serrano; Shaila García-Catalán; Marta Martín-Núñez
}

Cómo citar este artículo:

Rodríguez-Serrano, Aarón; García-Catalán, Shaila; Martín-Núñez, Marta (2019). “Estrategias narrativas audiovisuales de desinformación en YouTube de la nueva extrema derecha europea". El profesional de la información, v. 28, n. 3, e280311.

https://doi.org/10.3145/epi.2019.may.11

Artículo recibido el 08-01-2019 Aceptación definitiva: 01-05-2019

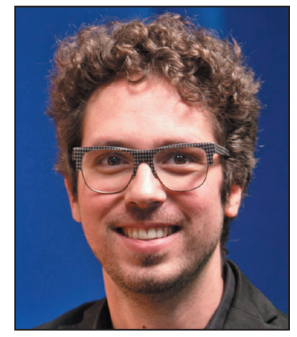

Aarón Rodríguez-Serrano $\square$ https://orcid.org/0000-0002-3858-1045

Universitat Jaume I

Facultad de Ciencias Humanas y Sociales Departamento de Comunicación

Avda. Sos Baynat, $\mathrm{s} / \mathrm{n}$.

12071 Castellón de la Plana, España

serranoa@uji.es

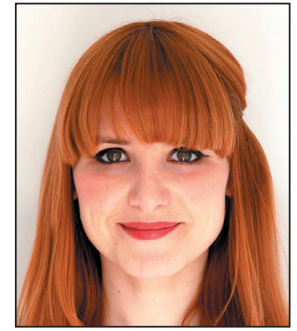

Shaila García-Catalán

https://orcid.org/0000-0003-2508-9091

Universitat Jaume I

Facultad de Ciencias Humanas y Sociales Departamento de Comunicación

Avda. Sos Baynat, s/n.

12071 Castellón de la Plana, España

scatalan@uji.es

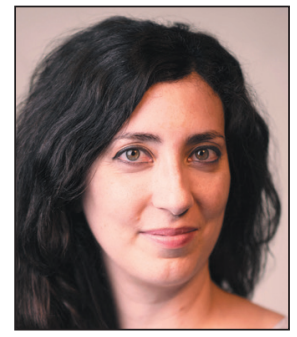
Marta Martín-Núñez
https://orcid.org/0000-0002-9473-1183
Universitat Jaume I
Facultad de Ciencias Humanas y Sociales
Departamento de Comunicación
Avda. Sos Baynat, s/n.
12071 Castellón de la Plana, España
mnunez@uji.es

\section{Resumen}

El presente trabajo analiza las estrategias de desinformación de 12 partidos europeos de extrema derecha mediante el análisis textual narratológico de los contenidos alojados en sus canales oficiales de YouTube. Para ello se analiza tanto el discurso del total de los videos alojados (12.961) como los procesos de significación precisos (puesta en escena, sistema de narradores...) sobre la muestra de los 36 clips más compartidos y visitados. Se concluye que la mayoría construye narraciones basadas en el odio, visualmente sustentadas sobre la figura de un único líder que apela (mediante un montaje analítico y la ralentización del metraje) a la resurrección de una comunidad privilegiada basada en la exclusión de los sujetos no afines.

Financiación

El presente trabajo ha sido realizado en el marco de los proyectos de investigación Análisis de identidades discursivas en la era de la posverdad. Generación de contenidos audiovisuales para una educomunicación crítica (Aidep) (código 181390.01/1) dirigido por el Dr. Javier Marzal-Felici; y El diseño narratológico en videojuegos: una propuesta de estructuras, estilos y elementos de creación narrativa de influencia postclásica (DiNaVi) (código 181369.01/1), dirigido por la Dra. Marta Martín-Núñez. Ambos fueron financiados por la Universitat Jaume I, a través de la convocatoria competitiva de proyectos de investigación de la UJI para el período 2019-2021. 


\title{
Palabras clave
}

Desinformación; Comunicación política; Partidos políticos; Extrema derecha; Racismo; Populismo; Narratología; Análisis textual; Redes sociales; YouTube; Europa.

\begin{abstract}
The disinformation strategies of 12 extreme right-wing European parties through the narrative textual analysis of the contents shared by their official YouTube channels are analyzed. In order to achieve our goal, we analyze in the first place the discourse of the total number of videos hosted $(12,961)$, and then, the precise meaning processes (staging, narrator system...) on a sample of the 36 most shared and visited clips. We show that the vast majority of the parties build discourses based on the hate, visually supported on the figure of a single leader who appeals (through an analytical montage and the slowing down of the footage) to the resurrection of a privileged community based on the exclusion of the external subjects.
\end{abstract}

\section{Keywords}

Disinformation; Political communication; Political parties; Far Right; Racism; Populism; Narratology; Textual analysis; Social media; YouTube; Europe.

\section{Introducción. La nueva extrema derecha en las redes}

Los buenos resultados obtenidos por algunas formaciones de extrema derecha en las elecciones europeas de mayo de 2014 colocaron el foco mediático en estos partidos, pese a que habían estado presentes en el mapa político europeo desde los años 80. Han sido descritos como "nueva extrema derecha" o "derecha radical populista" (Mudde, 2007) por su adaptación al sistema liberal para su supervivencia. Estos partidos han crecido en casi la totalidad de los países europeos apoyados en tres ejes: el ultranacionalismo, el populismo y el autoritarismo (Casals-Meseguer, 2003; Mudde, 2007; Antón-Mellón, 2007) en un contexto especialmente propicio por la crisis económica de 2008 y la inestabilidad en Siria e Irak. Esto ha centrado en dos temas principales su movilización política (aunque con ciertas especificidades nacionales) (Antón-Mellón; Hernández-Carr, 2016):

- discurso anti-establishment;

- rechazo a la población extranjera.

La mayor parte de los países de la Unión Europea tienen representación de la extrema derecha en sus parlamentos nacionales en estos momentos. España era, hasta las elecciones del 28 de abril de 2019, uno de los países europeos en los que aún no había penetrado, junto a Irlanda, Malta, Luxemburgo y Portugal.

Las elecciones andaluzas del 2 de diciembre de 2018 suponen un antes y un después en el mapa político español por la aparición de Vox, un partido que se sitúa ideológicamente en este espacio político, y que participa por primera vez en un parlamento autonómico español con 12 escaños. Su irrupción institucional se replica después, con más o menos intensidad, en las elecciones europeas, generales, autonómicas y municipales de 2019. A nivel europeo, pese a las diferencias existentes entre estos partidos - pues no han logrado conformar un grupo político en el Parlamento Europeo- sí tienen en común su aproximación de estilo populista para generar una amplia base social.

La bibliografía científica coincide en señalar la dificultad de definir el populismo (Taggart, 2000; Alvares; Dahlgreen, 2016), que ha ido mutando y se escapa a generalizaciones y definiciones, y que incluso puede llegar a operar como un "significante vacío" (Laclau, 2007). Sin embargo, existe una coincidencia en señalar que los partidos de la derecha radical populista plantean un reduccionismo demagógico para ofrecer soluciones simples a temas complejos señalando a un culpable (Pelinka, 2013). Los discursos políticos están adoptando un tono apocalíptico que afecta cada vez más a las concepciones contemporáneas del poder, recordando incluso a las interpretaciones del infierno de la Europa medieval (Hardt; Negri, 2011). Aunque el capitalismo cada vez necesita menos de la democracia, hace constantemente referencia en sus discursos a los valores democráticos como coartada para las estrategias neoliberales más feroces (Alemán, 2016). La demagogia y la ambigüedad son factores que atraviesan las barreras ideológicas y sociológicas (Antón-Mellón; Hernández-Carr, 2016) para influir en el comportamiento individual y colectivo. Y es que las sociedades contemporáneas se articulan como "comunidades de goce" (Palao-Errando, 2013) fundadas en la agresividad y no tanto en la consistencia simbólica.

En estos escenarios donde la comunicación se propone como único campo de enunciación de la política (García-Catalán, 2013), las plataformas digitales y las redes sociales juegan un importante papel en la producción, distribución y consumo de contenidos e información política (Casero-Ripollés, 2018). Los partidos de este espacio político trazan estrategias de comunicación para diseminar sus propuestas ideológicas, al margen de su presencia —en algunos casos aún minoritaria, cuestionada u obstaculizada - en los medios de comunicación tradicionales y en los foros públicos, como fue el caso del Front national francés (Ilamado Rassemblement national desde junio de 2018) (Bratten, 2005). 
El presente trabajo se aproxima a la construcción de los discursos audiovisuales de la derecha radical populista europea para analizar cuáles son sus estrategias narrativas de desinformación online en un contexto de crisis socioeconómica. Para ello nos centramos en el análisis de los contenidos generados explícitamente por los partidos con una intencionalidad de desinformación que se encuentran alojados en sus respectivos canales de vídeo de YouTube, que actúan como repositorio (a partir de los cuales lanzar los contenidos a otros medios) y como red social en sí misma, por sus propias posibilidades de gestionar suscriptores e interactuar con 'me gusta' y comentarios en los vídeos. Pese a que los vínculos entre los nuevos medios y la extrema derecha han sido ampliamente estudiados (Atton, 2006; Bratten, 2005; Cammaerts, 2009), creemos que este estudio puede ser relevante atendiendo a los retos de futuro que señala Casero-Ripollés (2018) sobre la investigación del impacto de las redes sociales en la producción, distribución y consumo de la información política.

La novedad de esta investigación radica en su aproximación desde la estrategia narrativa de la construcción de los mensajes audiovisuales con la intención de ir más allá de la descripción para analizar el cómo, el porqué y los efectos que estos discursos audiovisuales conllevan desde una visión crítica.

Del mismo modo pensamos que ofrecemos una primera aproximación que podría ser posteriormente completada desde campos paralelos al estudio narratológico como los estudios de recepción. Se trata de un estudio en el que dialogan estrategias de doce partidos de varios países europeos sin estar circunscrito a un proceso electoral concreto, lo que arroja resultados más amplios. Tenemos en cuenta también los vídeos más vistos en campaña electoral, especialmente aquellos que llegan a formar parte de los más populares a largo plazo (Berrocal-Gonzalo; Martín-Jiménez; Gil-Torres, 2017).

Por otra parte, la investigación en la plataforma YouTube es aún escasa (Berrocal-Gonzalo; Martín-Jiménez; Gil-Torres, 2017) frente a un predominio en esta área en torno a Twitter (Campos-Domínguez, 2017) pese a que se sitúa como la segunda red que más está cambiando el periodismo (Campos-Freire et al., 2016).

Los contenidos políticos no son los que gozan de mayor éxito en YouTube: los de entretenimiento tienen mayor frecuencia, número de visualizaciones e interacción de los usuarios. Quizás sea prematuro hablar de un autoabastecimiento informativo porque todavía no hay una significativa actividad creadora independiente de los medios de comunicación de masas (Rodríguez-Breijo; Gallardo-Camacho; Sierra-Sánchez, 2018). Como ocurre en Instagram (López-Rabadán; Doménech-Fabregat, 2018), la gestión política de YouTube se encuentra todavía en una fase de experimentación en busca de sus funciones más eficaces y un estilo comunicativo definitivo. Sin embargo YouTube es idóneo para lo que Castells denomina la autocomunicación de masas (2009). Esta plataforma permite un modelo más horizontal basado en la autogestión: los usuarios producen sus propios mensajes, su emisión es autogestionada, y los receptores seleccionan cuándo y cómo consumir los contenidos. Por ello cuando analizamos partidos minoritarios de ideología extremista, algunos sin representación parlamentaria nacional y que apenas reciben atención por parte de los principales medios de comunicación tradicionales, YouTube se convierte en una importante ventana para desplegar su ideología.

Del mapa que elabora Casero-Ripollés (2018) sobre investigación política y redes sociales, extraemos tres cuestiones que pueden facilitar la desinformación en los contenidos políticos que producen y distribuyen los propios partidos a través de plataformas como YouTube:

- Los actores políticos utilizan las plataformas digitales principalmente para difundir contenidos de auto-presentación y auto-expresión directamente a los usuarios, sin necesidad de que estos mensajes pasen por los medios de comunicación tradicionales, que pierden el control sobre lo que llega a la audiencia.

- En el entorno digital se ha impuesto un modelo que facilita el consumo casual de contenidos, por lo que no es necesaria una búsqueda de información política activa por parte de los usuarios. Los contenidos llegan a través de sus redes sociales de forma casual, lo que puede aumentar la polarización política y la radicalización ideológica.

- Las informaciones capaces de generar emociones pueden movilizar a gran cantidad de público hacia su consumo ya que los individuos aislados se articulan alrededor de apelaciones afectivas.

En nuestra cultura visual se extiende de forma decidida esa mirada cautiva(dora) del cine en la que la mostración domina a la narración (Company-Ramon; Marzal-Felici, 1999; Rodríguez-Serrano, 2017) y que deriva en la noción de infoentretenimiento tan presente en los vídeos políticos (Berrocal-Gonzalo; Martín-Jiménez; Gil-Torres, 2017). Esta construcción del espectáculo, que atiende más al discurso del exceso que a una narrativa sólida, se incrementa en los escenarios de la crisis de lo real propiciada por el impacto de la crisis económica y financiera de 2008 en la sociedad contemporánea (Marzal-Felici; Soler-Campillo, 2018).

En este panorama las plataformas digitales se configuran como espacios en los que los actores políticos contra-hegemónicos se convierten en actores comunicativos con un amplio margen de autonomía al poder producir y difundir sus propios contenidos activando procesos de automediación a través de las redes. Éstas se adecúan a las tendencias del complejo pensamiento político posmoderno que, contra el espectro del sujeto trascendental, intentan afirmar la proliferación liberadora de las múltiples formas de la subjetividad (femenina, homosexual, étnica) en las que importa más el reconocimiento social que la lucha socioeconómica (Žižek, 2007). Sin embargo, el modelo red ha servido tanto a los intereses de activistas políticos vinculados a movimientos sociales como el 15M o la Primavera Árabe (Casero-Ripollés, 2015) como a los grupos de extrema derecha (Cammaerts, 2009). 


\section{Objetivos}

Una vez desarrollado el marco teórico de nuestra investigación, podemos sistematizar los siguientes objetivos:

1. Ofrecer un mapa sistemático de la presencia oficial de la nueva extrema derecha más relevante en YouTube.

2. Sistematizar sus estrategias narrativas en una muestra representativa a propósito de los siguientes campos:

2a. Procesos de desinformación sobre la puesta en escena y la puesta en cuadro (trabajo estético del frame y de los movimientos de cámara).

2b. Procesos de desinformación sobre la puesta en serie (montaje).

2c. Procesos de desinformación mediante la construcción narratológica (tema, personaje, sistema de narradores, gestión del punto de vista...).

\section{Metodología. Muestra y procedimiento de recogida de datos ${ }^{1}$}

El diseño de la investigación se realizó en dos fases, correspondientes a los dos objetivos de investigación enunciados.

En la fase 1 (entre octubre y noviembre de 2018 y vinculada al objetivo de investigación 1) se realizó un estudio pormenorizado del mapa completo de las cuentas oficiales gestionadas por los partidos políticos de extrema derecha con representación parlamentaria. Más adelante, y debido a la irrupción de Vox en los comicios correspondientes a las elecciones andaluzas el 2 de diciembre de 2018, se consideró pertinente su inclusión en el universo, así como el estudio de sus propios mecanismos audiovisuales.

En esta primera fase se realizó una exploración general de los contenidos de los canales excluyendo del universo aquellos partidos que no tenían canal oficial de YouTube, aquellos que no generaban contenido propio sino que se limitaban a replicar noticias de los medios afines, y aquellos que estaban claramente desactualizados. En el caso de los países que contaban con más de una formación susceptible de ser considerada de extrema derecha (Hungría, Polonia o Bélgica) se estudió aquella con mayores seguidores y mayor relevancia parlamentaria. Este primer filtro nos permitió acotar nuestra búsqueda a 12 partidos que habían nutrido sus plataformas con un total de 12.961 videos. Finalmente, la fase 1 arrojó la muestra que se recoge en la tabla 1, con datos actualizados a 7 de enero de 2019.

Tabla 1. Canales y contenidos de los doce países estudiados

\begin{tabular}{|l|l|c|c|}
\hline \multicolumn{1}{|c|}{ País } & \multicolumn{1}{|c|}{ Partido } & Videos subidos & Seguidores YouTube \\
\hline Hungría & Jobbik & 2.354 & 12.674 \\
\hline Polonia & Ley Justicia & 478 & 7.780 \\
\hline Austria & Partido de la Libertad & 1.689 & 22.722 \\
\hline Francia & Frente Nacional & 79 & 24.499 \\
\hline Dinamarca & Partido Popular Danés & 19 & 144 \\
\hline Reino Unido & UKIP & 129 & 13.011 \\
\hline Alemania & Alternativa para Alemania & 771 & 26.453 \\
\hline Holanda & Partido de la Libertad & 210 & No disponible \\
\hline Grecia & Amanecer Dorado & 1.067 & No disponible \\
\hline España & Vox & 726 & 70.053 \\
\hline Suecia & Demócratas suecos & 314 & 24.790 \\
\hline Italia & Lega Italia & 5.125 & 21.784 \\
\hline
\end{tabular}

Esta primera aproximación ya muestra varias características del objeto de estudio sobre las que parece necesario detenerse. Si bien es relevante para intuir el tamaño concreto de cada uno de los canales, se trata de una muestra heterogénea en cuanto a actividad, volumen y clasificación de los materiales. La monitorización de seguidores de la cuenta de Vox permite apreciar que es el partido con mayor número de seguidores en YouTube de toda Europa: pasó de cerca de 44.000 suscriptores a 61.000 en las dos semanas posteriores a su irrupción en el parlamento de Andalucía, contaba con 70.053 en enero de 2019 (tabla 1) y llegó a los 157.000 en las seis semanas posteriores a las elecciones generales del 28A (2019). Por el contrario la cuenta de Amanecer Dorado fue cancelada por YouTube en diciembre de 2018 y se encuentra en proceso de reconstrucción. Sobre esta primera muestra se estableció un segundo filtro basado en el análisis de contenido del universo que Vox pasó de 61.000 suscriptores en YouTube en las dos semanas posteriores a su irrupción en el parlamento de Andalucía a 157.000 en las seis semanas posteriores a las elecciones generales del $28 \mathrm{~A}$

quedará explicitado en el epígrafe 3.1 y que permitió conocer con más detalle:

- los materiales concretos (entrevistas, reportajes, intervenciones parlamentarias...);

- su origen (creado por el propio partido o recogido de los medios de comunicación afines);

- sus intereses ideológicos concretos. 
Una vez trazado el mapa completo, la fase 2 (entre noviembre y diciembre de 2018 y vinculada al objetivo de investigación 2) consistió en el análisis textual y narratológico exhaustivo de una serie de unidades de contexto representativas de cada canal. Se tomaron como muestra tres vídeos de cada canal tomando como referencia el número de visitas y el hecho de que se tratara de contenidos específicamente diseñados para su transmisión a partir de YouTube. Se contó así con 36 vídeos con un total de 5.115 minutos de duración repartidos en 1.473 planos.

El análisis de la fase 2 se realizó con herramientas precisas derivadas del estudio narratológico de los discursos (Bal, 2017), especialmente teniendo en cuenta sus dimensiones retóricas (Kearns, 1999) y afectivas (Colm-Hogan, 2011). Para ello se ha refinado la herramienta desarrollada por Ekman (2014) con la aplicación sistemática de dos modelos de análisis textual plenamente asentados:

- la tripartición de elementos narrativos audiovisuales de Gómez-Tarín (2016) -correspondiente a las áreas II, III y IV de la herramienta-;

- el sistema clásico de focalización y gestión de punto de vista/narradores teorizado por Gaudreault y Jost (1995) -correspondiente al bloque $\mathrm{V}$.

El diseño final queda recogido en la tabla 02.
Tabla 2. Herramienta principal de análisis narratológico

\begin{tabular}{|c|c|}
\hline Área & Ítem medido \\
\hline \multirow{4}{*}{ I. Identificación } & 01. Partido al que pertenece \\
\hline & 02. Duración (en segundos) \\
\hline & 03. Duración (en planos) \\
\hline & 04. Duración (en secuencias) \\
\hline \multirow{6}{*}{ II. Puesta en escena } & 01. Personaje principal \\
\hline & 02. Personajes secundarios \\
\hline & 03. Localización \\
\hline & 04. Ambientación \\
\hline & 05. Iluminación \\
\hline & 06. Efectos digitales añadidos \\
\hline \multirow{4}{*}{ III. Puesta en cuadro } & 01. Escala de plano \\
\hline & 02. Angulación de plano \\
\hline & 03. Movimiento de cámara \\
\hline & 04. Composición compleja \\
\hline \multirow{6}{*}{ IV. Puesta en serie } & 01. Tipo de montaje \\
\hline & 02. Uso de montaje \\
\hline & 03. Uso del sonido \\
\hline & 04. Uso de la voz \\
\hline & 05. Uso de la música \\
\hline & 06. Aceleración/ralentización \\
\hline \multirow{5}{*}{$\begin{array}{l}\text { V. Elementos constructivos } \\
\text { del relato }\end{array}$} & 01. Sistema de narradores \\
\hline & 02. Diseño temporal \\
\hline & 03. Diseño estructural \\
\hline & 04. Función significante principal \\
\hline & 05. Relevancia de los actores políticos \\
\hline
\end{tabular}

La división en estas cuatro áreas nos garantiza una cobertura completa sobre aquellos elementos que componen la forma audiovisual y, por lo tanto, como ya quedó demostrado al hilo de la propaganda fílmica nazi (Zumalde-Arregi, 2007), en los que se juegan realmente los procesos de significación política. Tanto en la fase 1 como en la fase 2 se aplicaron métodos de estadística descriptiva mediante el programa SPSS v. 24.

\section{Resultados}

\subsection{La extrema derecha europea en YouTube: materiales, origen e intereses ideológicos}

Como se ha señalado anteriormente, la clasificación, consumo y fidelización de los canales analizados mostraba una naturaleza extraordinariamente heterogénea y en rápido cambio, que resultaba poco fiable como posible punto de partida. En su lugar, el análisis de contenido de cada uno de los canales ha permitido comprender con mayor precisión cómo funcionan los mecanismos de desinformación y a qué temas y colectivos afectan.

Sobre el origen de los materiales, se constata que un $66 \%(\mathrm{~N}=8)$ de la muestra opta por nutrir su canal con piezas generadas desde los medios de comunicación tradicionales de su país. Obviamente se trata siempre de plataformas afines ideológicamente que han registrado algún tipo de intervención del equipo político o han tratado con un enfoque claramente laudatorio sus presentaciones públicas o los actos sociales propios del partido.

Centrándonos en esos ocho partidos concretos que se nutren de material ajeno, el desglose concreto de su naturaleza queda recogido en la tabla 3.

Tabla 3. Naturaleza de los materiales externos

\begin{tabular}{|l|c|c|}
\hline \multicolumn{1}{|c|}{ Naturaleza de los materiales externos } & N. de partidos que comparten estos materiales & 7 \\
\hline Entrevistas al líder & 58,3 \\
\hline Entrevistas a otros miembros del partido político & 6 & 2 \\
\hline Entrevistas a militantes del partido & 30,0 \\
\hline Reportajes extraídos de la televisión nacional & 3 & 25,0 \\
\hline Entrevistas grabadas en video de emisiones radiofónicas & 3 & 25,0 \\
\hline
\end{tabular}


El género privilegiado es la entrevista, especialmente cuando se centra en el líder principal del partido. Como veremos más adelante, será un dato que coincidirá con el estudio narratológico de las piezas específicamente propagandísticas. El militante del partido queda relegado a un segundo lugar y sus intervenciones son siempre presentadas desde una perspectiva laudatoria, a menudo sintetizadas en frases breves y concisas que coinciden inevitablemente con los puntos del programa de turno.

El género reportaje es menos reconocido y cuando se utiliza suele tomar tintes bufonescos o paródicos. A modo de ejemplo, Lega Italia comparte un reportaje emitido por Tele5 Italia titulado Ecco cosa pensano le donne musulmane en el que se ridiculizan las ropas, tradiciones y los acentos de mujeres inmigrantes de los países árabes.

https://www.youtube.com/watch?v=K-5rKIAd-Yc

Otro detalle que merece la pena señalar es el auge indudable de las entrevistas radiofónicas grabadas, que en casos como Vox pueden llegar a nutrir prácticamente una lista completa (Vox en los medios) compuesta por más de quinientas emisiones.

A continuación, consideraremos los aspectos ideológicos concretos que configuran el discurso desinformativo. Podemos destacar que todos los partidos estudiados optan por un enfoque xenófobo, situando a la inmigración como el problema principal de la situación europea. El fundamentalismo islámico aparece citado en un $83,3 \%$ de los casos ( $\mathrm{N}=10)$ como el enemigo explícito, seguido muy de lejos por las referencias al judaísmo $(16,7 \% \mathrm{~N}=2)$ y, en el caso concreto del UKIP británico, contra la inmigración de los países del Este de Europa. La fundamentación del discurso en elementos islamófobos cuenta con toda una serie de matices perversos sobre los que merecería la pena detenerse: desde la ya citada ridiculización por la Lega italiana, a la apropiación de videos del Estado Islámico por parte de los ultraderechistas suecos, hasta la campaña basada en el terror del Partido por la libertad holandés, que identifica en bloque a todo el Islam con el terror, la opresión, la violencia y la conquista.

En cuanto a las causas secundarias que ofrecen para explicar la hipotética crisis de valores y de identidad de sus respectivos países - además de la inevitable situación de inestabilidad económica一, se observa una mayor disparidad en los discursos. Un $58,3 \%$ de los partidos $(\mathrm{N}=7$ ) se muestra opuesto a la permanencia de sus respectivos países en la Unión Europea - si bien, paradójicamente, todos los que cuentan con representación en el Parlamento Europeo cuelgan en su canal los videos con las intervenciones de sus líderes y diputados. El mismo porcentaje sugiere la supremacía nacional, apelando a las raíces identitarias del pueblo, a su capacidad productiva o a las bases de cooperación entre ciudadanos del mismo país. Un porcentaje algo mayor $(75 \%, \mathrm{~N}=9)$ hace referencia al reparto desigual de la riqueza entre los países de la zona europea, si bien únicamente un $41,7 \%(\mathrm{~N}=5)$ incorpora mensajes contra el capitalismo o el neoliberalismo. Sin embargo la culpa del mal estado económico de la población suele achacarse de nuevo a la inmigración o a la mala gestión de aquellos partidos liberales a los que sistemáticamente se acusa de traicionar y engañar a sus ciudadanos — por supuesto, en clara connivencia con los medios de comunicación tradicionales.

Algo más se puede añadir sobre la fundamentación ideológica del mensaje supremacista nacional: un $33 \%(\mathrm{~N}=4)$ basa su discurso en la mención más o menos explícita de las gestas militares del pasado -en su gran mayoría, conquistadores y altos mandos de noble tradición histórica, y/o en la fuerza de la religión compartida como guía en el proceso reconstructor de los valores nacionales perdidos. Un 41,7\% ( $\mathrm{N}=5)$ menciona también la familia nuclear tradicional heteronormativa como elemento social garante del buen funcionamiento del país y elemento privilegiado de su programa discursivo.

A continuación se detalla cómo se transmiten estos mensajes mediante el análisis narratológico-audiovisual de los videos concretos.

\subsection{Elementos de puesta en escena}

El desglose principal de la puesta en escena - como en el resto de campos narratológicos- suele venir dado en función de la correcta transmisión del mensaje prioritario: construir audiovisualmente la seducción que emerge del líder. Esto resulta evidente al contemplar el desglose de los personajes principales que domina la muestra seleccionada que se recoge en la tabla 4.
Tabla 4. Frecuencia de los protagonistas de la puesta en escena

\begin{tabular}{|l|c|c|}
\hline \multicolumn{1}{|c|}{ Protagonistas de la puesta en escena } & Frecuencia & $\%$ \\
\hline El líder & 16 & 46,8 \\
\hline El equipo de gobierno & 1 & 2,8 \\
\hline Los militantes & 7 & 19,4 \\
\hline Los posibles votantes & 5 & 13,9 \\
\hline El partido como unidad & 2 & 5,6 \\
\hline No se detecta un personaje principal & 4 & 11,1 \\
\hline
\end{tabular}

Resulta llamativo que el líder concreto de cada partido sea situado en el centro del discurso, en detrimento de sus propios compañeros de formación o de la propia marca política. Como suele ocurrir en la fundamentación de los discursos ultraderechistas, se privilegia la identificación entre partido, líder y nación (Spotts, 2011) a partir de una serie de efectos afec- 
tivos básicos que analizaremos más adelante. Respecto al número de planos concretos destinados al líder, del total de 1.473 planos analizados, el 31,5\% ( $N=564)$ se dedica a retratar al miembro principal de la formación. En algunos casos este porcentaje se dispara, como por ejemplo en los vídeos dedicados a Marine Le Pen - que interviene en un $72 \%(\mathrm{~N}=113)$ de la muestra- o de Santiago Abascal - presente en un 79,3\% ( $\mathrm{N}=127)$ de los planos analizados del canal de Vox.

En cuanto a la composición del profílmico, se detecta una clara preferencia por las localizaciones urbanas $(61,1 \%, \mathrm{~N}=22)$, iluminadas en clave alta $(63,9 \%, N=23)$ y generalmente rubricadas por algún tipo de efecto digital tipográfico $(47,2 \%$, $\mathrm{N}=17$ ). Hay de nuevo excepciones y matices. Los Demócratas suecos, por ejemplo, priorizan los planos nocturnos reforzados por la iluminación en clave baja propios de la dirección de fotografía del género negro nórdico popularizada por nombres como Eric Kress, Dion Beebe o Hoyte Van Hoytema. También resulta llamativa la manera en la que —al contrario de lo que ocurre en la propaganda escrita de corte más extremo (Schuppener, 2015) - las localizaciones han tendido a separarse de la Heimat (país, hogar) nacionalsocialista para optar principalmente por escenarios urbanos y la descripción de oficios vinculados con la construcción, la tecnología o el servicio.

\subsection{Elementos de puesta en cuadro y puesta en serie}

El trabajo sobre la angulación y el movimiento de la cámara también arroja algunos datos relevantes sobre la construcción del discurso. En primer lugar, se privilegia la exhibición de los espacios mediante el plano general ( $N=385$ ) y de los cuerpos mediante el plano medio corto ( $\mathrm{N}=456)$. Resulta muy interesante, especialmente en la construcción de las figuras de poder, un menor recurso a los primeros planos $(\mathrm{N}=130)$ y un todavía inferior uso de los primerísimos primeros planos ( $\mathrm{N}=48$ ). Parece que se impone una cierta distancia enunciativa en el ejercicio de la mostración, una espacio respetuoso que reserva la cercanía únicamente para aquellos momentos de especial emotividad o que parecen exigir un suplemento melodramático. Salvo aquellos (escasos) líderes que se muestran a sí mismos en actividad relajada o incluso desenfadada —es el caso del austríaco Heinz-Christian Strache a lo largo de su colección de particulares raps-, la cámara suele buscar momentos de intensidad dramática, generalmente mediante la forma de planos medios ralentizados con una ligera inclinación en contrapicado (angulación oblicua inferior de la cámara, hacia arriba), que retratan su actividad exclusivamente salvadora: rodeados de masas de militantes, baños de banderas o en sobrecogedores parajes naturales. De nuevo la cámara se sigue mostrando deudora de las huellas estilísticas esbozadas durante los célebres primeros minutos —el descenso mesiánico del Führer (Sennett, 2014) - de El triunfo de la voluntad (Triumph des Willens, Leni Riefenstahl, 1935).

En cuanto al movimiento y angulación de cámara, la enunciación privilegia los travellings ( $\mathrm{N}=149$ ) frente a las panorámicas $(\mathrm{N}=44)$, y los planos aéreos $(\mathrm{N}=75)$ frente a los contrapicados $(\mathrm{N}=57)$. El primer caso es fácilmente comprensible a la luz del abaratamiento de los equipos tecnológicos que garantizan una mayor estabilización de la imagen y que han acabado por reducir la panorámica a un gesto más televisivo que cinematográfico - y, por lo tanto, más dado a la información que a la épica. En cuanto al segundo dato, llama la atención que en la mayoría de los casos la angulación aérea se combina con la escala general, dotando a los exteriores naturales o urbanos de una espectacularización extraordinaria sobre la que se suele recortar de manera épica la figura del líder del partido.

Finalmente, los datos referentes al montaje muestran una notable cohesión interna. Un 75\% de los vídeos optan por un montaje de tipo analítico, sin que se haya podido encontrar ningún ejemplo notable de fallo de raccord o de continuidad. Lo más cercano que se puede apreciar a este tipo de huellas enunciativas son algunos anecdóticos saltos del eje óptico para reencuadrar personajes. Es interesante también que en términos narrativos, el montaje opta en la mayoría de los casos $(61,1 \%, \mathrm{~N}=22)$ por un efecto discursivo frente a una menor ordenación narrativa $(38,9 \%, \mathrm{~N}=14)$, que generalmente suele reducirse a los testimoniales dramatizados del líder — por ejemplo, la visita protagonizada por Marine Le Pen a los caídos de la Primera Guerra Mundial en el vídeo de presentación de su canal. Es llamativo que los recursos dramáticos de postproducción más utilizados sean la música extradiegética -música introducida en la postproducción- $(80,6 \%, N=29)$ y la ralentización del plano (316 planos ralentizados, esto es, un 21,4\% del total de planos sometidos a análisis). Lógicamente nos encontramos de nuevo ante una voluntaria espectacularización del gesto político que busca forzar la transmisión de mensajes mediante la conjuración de un trayecto heroico apocalíptico. Por ejemplo, el plano detalle ralentizado con música épica de la mano del líder acariciando la flora nacional se repite sistemáticamente, citando así el imaginario concreto desatado por Gladiator (Ridley Scott, 2000), cinta directamente fundamentada sobre la construcción audiovisual del III Reich.

\subsection{Construcción del relato desinformativo}

Llegados a este punto podemos finalmente concretar cómo estos recursos audiovisuales se hilvanan en un proceso de desinformación concreto. Para ello, en primer lugar se ha estudiado el tema principal que emerge del análisis crítico del discurso que recoge la tabla 5.

Como se puede apreciar, reaparece de nuevo el hecho de generar un "otro" antagónico al que odiar - y al que, a juzgar por las cifras, únicamente el líder está en condiciones de rendir batalla. Si atendemos a los elementos narratológicos analizados en términos de operadores textuales significantes concretos — música, angulación de cámara, montaje...- vemos 
que hasta un $22 \%$ de los videos estudiados $(\mathrm{N}=8)$ buscan abiertamente infundir el terror y la inseguridad en el espectador. Es el caso de la música extradiegética death metal que viste los desfiles paramilitares de los miembros de Amanecer Dorado o los planos aéreos ralentizados que dan cuenta de la llegada de inmigrantes a Europa desde los países árabes en los videos de Alternative für Deutschland entre otros. Únicamente se han detectado 3 vídeos (8,3\%) en los que el partido ofrece como evidencias datos estadísticos concretos, verificables y ajenos a sus propias fuentes. Resulta evidente que los intereses informativos están muy lejos de los discursos que proponen en esta plataforma digital.

No extraña, por tanto, que una gran cantidad de vídeos $(\mathrm{N}=21,58,3 \%)$ se valgan de narradores subjetivos y diegéticos (se hallan en el espacio fílmico formando parte de la historia). Además, en muchas ocasiones ( $\mathrm{N}=14,38 \%)$ es el propio líder el que marca su agenda mediante una voz en off que transmite, de manera irremediable y estrictamente lineal - la cronología del relato se mantiene en un $66,7 \%$ de los videos-, los mismos mensajes: amenazas del exterior, crisis de valores, ciudadanía engañada por políticos corruptos, ante los cuales, él o ella son la única excepción posible y, finalmente, posibilidad de una redención patriótica, excluyente y nacional.

\section{Conclusiones}

Volviendo a los objetivos iniciales, sintetizamos los siguientes resultados.

R1: La extrema derecha europea oficial cuenta en YouTube con una amplia base de seguidores que aglutina casi 13.000 vídeos en diferentes canales que tienden a generar su propio contenido o, en menor medida, a nutrirse de los contenidos de los medios de comunicación tradicionales afines. El enfoque ideológico principal de los contenidos orbita mayoritariamente alrededor de la islamofobia, y en menor medida, del antieuropeismo. Domina el discurso del odio al otro.

R2a: El trabajo de cámara suele ponerse al servicio de una correcta exhibición del líder del partido, en oposición a la exhibición del equipo político, los militantes o los posibles votantes. Se suele situar su figura en escenarios exteriores, urbanos e iluminados en clave alta. Esto confirma la personalización de la política (Casero-Ripollés, 2012) que caracteriza el infoentretenimiento.

R2b: Se privilegian los planos generales y los planos medios para guardar una correcta distancia simbólica con la exhibición del líder, haciendo especial hincapié en los planos aéreos que dan grandeza a los espacios exteriores o que referencian fenómenos atmosféricos simbólicos vinculados al partido (tormentas, amaneceres y anocheceres...). Del mismo modo, los vídeos suelen funcionar mediante montaje analítico, utilizando especialmente dos recursos propios de la postproducción: la ralentización de planos y la introducción de música épica extradiegética que califican al líder de heroico.

R2c: El relato suele buscar generar enemigos comunes para la ciudadanía mediante herramientas significantes visualmente agresivas para dar consistencia más imaginaria que simbólica a una comunidad de goce. Por ello la gestión del punto de vista suele recaer en el líder del partido, que funciona además mediante la voz en off como narrador subjetivo de su propia historia.

Como hemos creído demostrar, el aprovechamiento de las nuevas tecnologías por parte de los grupos de extrema derecha no modifica en gran medida los rasgos narratológicos básicos que llevan construyendo la identidad fascista o incluso totalitarista desde sus comienzos:

- el culto cohesionador en la figura del líder (Sánchez-Biosca, 2006),

- la creación de un enemigo identitario sobre el que proyectar un discurso en el que se sugiera la exclusión o el exterminio (Rodríguez-Serrano, 2012),

- la unificación de las subjetividades de las masas en torno a categorías agraviadas y representadas mediante planos generales en picado (angulación oblicua de la cámara, mirando hacia abajo).

El trabajo visual funciona mediante el simulacro de un cierto "estado de la cultura" (Revert, 2017) en el que se profundiza en la emergencia, la crisis y, en el límite, el inminente riesgo de desaparición. El núcleo significante principal sigue siendo el miedo, la desazón, la afectividad, y casi nunca el desarrollo de acciones políticas concretas con un contenido proactivo, ni mucho menos constructivo.

Mientras que los materiales reciclados de medios de comunicación afines buscan una legitimación de su discurso en el tablero político democrático, el tratamiento de sus propias imágenes deja a las claras que su posición desconfía del resto de agentes políticos -incluyendo, por supuesto, a los medios de comunicación "no alineados", a los que generalmente se tiende a mostrar como cómplices de los desastres de la ciudadanía-. Se apoya más bien un discurso que prescinde de la razón para enraizarse en destinaciones históricas, misiones mesiánicas y, en el fondo, un profundo desprecio por la internacionalidad y el humanismo. 
Sin duda, la capacidad simbólica de los movimientos pro-democracia (Feenstra, 2017) tendrá que acometer uno de sus mayores retos: construir relatos cohesionadores que no se fundamenten en la exclusión y el miedo, sino en la posibilidad de generar una ciudadanía compatible en sus diferencias.

\section{Referencias}

Alemán, Jorge (2016). Horizontes neoliberales en la subjetividad. Buenos Aires: Grama. ISBN: 9789871982851

Alvares, Claudia; Dahlgreen, Peter (2016). “Populism, extremism and media: Mapping an uncertain terrain”. European journal of communication, v. 32, n. 1, pp. 46-57.

https://doi.org/10.1177/0267323115614485

Antón-Mellón, Joan (2007). “La cultura e ideología política del neopopulismo en Europa occidental: MNR/FN (Francia), FPÖ (Austria) y Lega Nord (Italia)". En: Simón, Miguel-Ángel (ed.). La extrema derecha en Europa desde 1945 a nuestros días. Madrid: Tecnos, pp. 281-310. ISBN: 9788430945108

Antón-Mellón, Joan; Hernández-Carr, Aitor (2016). “El crecimiento electoral de la derecha radical populista en Europa: parámetros ideológicos y motivaciones sociales". Política y sociedad, v. 53, n. 1, pp. 17-28.

https://revistas.ucm.es/index.php/POSO/article/view/48456

Atton, Chris (2006). "Far-right media on the internet: Culture, discourse and power". New media \& society, v. 8, n. 4, pp. 573-587.

https://doi.org/10.1177/1461444806065653

Bal, Michael (2017). Narratology: Introduction to the theory of narrative. Canada: Toronto Buffalo. ISBN: 9781442628342

Berrocal-Gonzalo, Salomé; Martín-Jiménez, Virginia; Gil-Torres, Alicia (2017). “Líderes políticos en YouTube: información y politainment en las elecciones generales de 2016 (26J) en España”. El profesional de la información, v. 26, n. 5, pp. $937-946$. https://doi.org/10.3145/epi.2017.sep.15

Bratten, L. Clare (2005) “Online zealotry: La France du peuple virtuel”. New media \& society, v. 7, n. 4, pp. 517-532. https://doi.org/10.1177/1461444805054116

Cammaerts, Bart (2009). "Radical pluralism and free speech in online public spaces: The case of North Belgian extreme right discourses". International journal of cultural studies, v. 12, n. 6, pp. 555-575.

https://doi.org/10.1177/1367877909342479

Campos-Domínguez, Eva (2017). "Twitter y la comunicación política”. El profesional de la información, v. 26, n. 5, pp.785-793. https://doi.org/10.3145/epi.2017.sep.01

Campos-Freire, Francisco; Rúas-Araújo, José; López-García, Xosé; Martínez-Fernández, Valentín-Alejandro (2016). "Impacto de las redes sociales en el periodismo". El profesional de la información, v. 25, n. 3, pp. 449-457. https://doi.org/10.3145/epi.2016.may.15

Casals-Meseguer, Xavier (2003). Ultrapatriotas. Extrema derecha y nacionalismo. De la guerra fría a la era de la globalización. Barcelona: Crítica. ISBN 9788484324300

Casero-Ripollés, Andreu (2012). “El periodismo político en España: algunas características definitorias”. En: Casero-Ripollés, Andreu (ed.). Periodismo político en España: concepciones, tensiones y elecciones. Tenerife: Cuadernos Artesanos de Latina, pp. 19-46.

http://repositori.uji.es/xmlui/handle/10234/80553

Casero-Ripollés, Andreu (2015). “Estrategias y prácticas comunicativas del activismo político en las redes sociales en España". Historia y comunicación social, v. 20, n. 2, pp. 533-548.

https://doi.org/10.5209/rev_HICS.2015.v20.n2.51399

Casero-Ripollés, Andreu (2018). "Research on political information and social media: Key points and challenges for the future". El profesional de la información, v. 27, n. 5, pp. 964-974.

https://doi.org/10.3145/epi.2018.sep.01

Castells, Manuel (2009). Comunicación y poder. Madrid: Alianza. ISBN 9788420684994

Colm-Hogan, Patrick (2011). Affective narratology: The emotional structure of stories. Nebraska: Nebraska University Press. ISBN 9780803230026

Company-Ramon, Juan-Miguel; Marzal-Felici, José-Javier (1999). La mirada cautiva. Formas de ver en el cine contemporáneo. Valencia: Generalitat Valenciana. ISBN: 8448222563.

Ekman, Mattias (2014). "The dark side of online activism: Swedish right-wing extremist video activism on YouTube". MedieKultur, v. 30, n. 56, pp. 79-99.

https://doi.org/10.7146/mediekultur.v30i56.8967 
Feenstra, Ramón A. (2017). "Rethinking global civil society and the public sphere in the age of pro-democracy movements". Journal of civil society, v. 13, n. 3, pp. 337-348.

https://doi.org/10.1080/17448689.2017.1359886

García-Catalán, Shaila (2013). “La política, el ladrón, su mujer y su amante”. En: Palao-Errando, José-Antonio (ed.). Política ( ) psicoanálisis. Cinco textos para inventar un vínculo. Santander: Shangrila Textos Aparte.

http://textosred.blogspot.com/2013/11/politica-psicoanalisis-cinco-textos.html

Gaudreault, André; Jost, François (1995). El relato cinematográfico: cine y narratología. Barcelona: Paidós. ISBN: 84 49300924

Gómez-Tarín, Francisco-Javier (2016). Elementos de narrativa audiovisual: expresión y narración. Cantabria: Shangrila. ISBN: 9788493828127

Hardt, Michael; Negri, Antonio (2011). Common Wealth. El proyecto de una revolución del común. Madrid: Akal. ISBN: 9788446032304

Kearns, Michael (1999). Rethorical narratology. Nebraska: Nebraska University Press. ISBN 9780803227422

Laclau, Ernesto (2007). On populist reason. London: Verso. ISBN: 9781788731317

López-Rabadán, Pablo; Doménech-Fabregat, Hugo (2018). “Instagram y la espectacularización de las crisis políticas. Las 5 W de la imagen digital en el proceso independentista de Cataluña”. El profesional de la información, v. 27, n. 5, pp.1013-1029.

https://doi.org/10.3145/epi.2018.sep.06

Marzal-Felici, Javier; Soler-Campillo, Maria (2018). “El espectáculo del exceso. Representaciones de la crisis financiera de 2008 en el cine mainstream norteamericano". Revista latina de comunicación social, n. 73, pp. 89-114. https://doi.org/10.4185/RLCS-2018-1247

Mudde, Cas (2007). Populist radical right parties in Europe. Cambridge: Cambridge University Press. ISBN: 9780521 850810

Palao-Errando, José Antonio (ed.) (2013). Política ( ) psicoanálisis. Cinco textos para inventar el vínculo. Santander: Shangrila Textos Aparte.

Pelinka, Anton (2013). "Right-wing populism: Concept and typology". In: Wodak, Ruth; Khosravinik, Majid; Mral, Brigitte (eds.). Right-wing populism in Europe. Politics and discourse. London: Bloomsbury, pp. 3-22. ISBN: 9781780932453

Revert, Jordi (2017). “Después del crímen perfecto: el futuro de la cultura en la era del simulacro". Vivat academia, v. 138, pp. 37-55.

https://doi.org/10.15178/va.2017.138.37-55

Rodríguez-Breijo, Vanessa; Gallardo-Camacho, Jorge; Sierra-Sánchez, Javier (2018). “Información política en los vídeos que son tendencia en YouTube España”. El profesional de la información, v. 27, n. 5, pp. 1041-1049.

https://doi.org/10.3145/epi.2018.sep.08

Rodríguez-Serrano, Aarón (2012). "La construcción cinematográfica del odio: las películas antisemitas del régimen nazi”. Revista de occidente, v. 373, pp. 113-126.

Rodríguez-Serrano, Aarón (2017). “Narrativa audiovisual, ontología y terrorismo: paradojas comunicativas en los videos del Estado Islámico". Palabra clave, v. 20, n. 1, pp. 96-115.

http://dx.doi.org/10.5294/pacla.2017.20.1.5

Sánchez-Biosca, Vicente (2006). Cine de historia, cine de memoria. La representación y sus límites. Madrid: Cátedra. ISBN: 9788437623146

Schuppener, George (2015). "Heimat und dialekt in rechtsextremer internet-kommunikation". Slowakische zeitschrift für Germanistik, n. 7, pp. 117-122.

http://www.sung.sk/fotky10204/SZfG/2015_1/117_PDFsam_SZfG_2015_1.pdf

Sennett, Alan (2014). "Film propaganda: Triumph of the Will as a case study". Framework: The journal of cinema and media, v. 55, n. 1, pp. 45-65.

https://muse.jhu.edu/article/556146/pdf

Spotts, Frederic (2011). Hitler y el poder de la estética. Madrid: Antonio Machado. ISBN: 9788477744498

Taggart, Paul A. (2000). Populism. Buckingham: Open University Press. ISBN: 9780335200467

Žižek, Slavoj (2007). El espinoso sujeto. El centro ausente de la ontología política. Buenos Aires: Paidós. ISBN: 978950 1265200

Zumalde-Arregi, Imanol (2007). La materialidad de la forma fílmica. Crítica de la (sin)razón posestructuralista. Bilbao: Universidad del País Vasco. ISBN: 978848377887 